\title{
Short popular review of quantum electromagnetodynamics
}

\author{
Rainer W. Kühne \\ kuehne@theorie.physik.uni-wuppertal.de
}

\begin{abstract}
The aim of this note is to give a short and popular review of the ideas which led to my model of magnetic monopoles and my prediction of the second kind of electromagnetic radiation. I will also point out the many and far-reaching consequences if these magnetic photon rays would be confirmed.
\end{abstract}

The discovery of a second kind of light would be a multi-dimensional scientific revolution. It would shake the foundations of modern physics in many ways. It would be experimental evidence of physics beyond the standard theory of particle physics. The standard theory includes the Weinberg-Salam theory from 1967/1968 and quantum chromodynamics from 1973. The observation would require not only that the theory of quantum electrodynamics formulated in 1948/1949 has to be extended. It would challenge also the Copenhagen interpretation of quantum mechanics formulated in 1927/1928. Furthermore, the new kind of light would violate the relativity principle of special relativity from 1905 and would require a symmetrization of Maxwell's equations from 1873.

The existence of the second kind of light was predicted theoretically. It can be understood by the following argumentation.

In 1948/1949 Tomonaga, Schwinger, and Feynman introduced quantum electrodynamics. It is the quantum field theory of electric and magnetic phenomena. This theory has one shortcoming. It cannot explain why electric charge is quantized, i.e. why it appears only in discrete units.

In 1931 Dirac introduced the concept of magnetic monopoles. He has shown that any theory which includes magnetic monopoles requires the quantization of electric charge.

A theory of electric and magnetic phenomena which includes Dirac monopoles can be formulated in a manifestly covariant and symmetrical way if two four-potentials are used. Cabibbo and Ferrari in 1962 were the first to formulate such a theory. Within the framework of a quantum field theory one four-potential corresponds to Einstein's electric photon from 1905 and the other four-potential corresponds to Salam's magnetic photon from 1966.

In 1997 I have shown that the Lorentz force between an electric charge and a magnetic charge can be generated as follows [1]. An electric charge couples via the well-known vector coupling with an electric photon and via a new type of tensor coupling, named velocity coupling, with a magnetic photon. This velocity coupling requires the existence of a velocity operator.

For scattering processes this velocity is the relative velocity between the electric charge and the magnetic charge just before the scattering. For emission and absorption processes there is no possibility of a relative velocity. The velocity is the absolute velocity of the electric charge just before the reaction. 
The absolute velocity of a terrestrial laboratory was measured by the dipole anisotropy of the cosmic microwave background radiation. This radiation was detected in 1965 by Penzias and Wilson, its dipole anisotropy was detected in 1977 by Smoot, Gorenstein, and Muller. The mean value of the laboratory's absolute velocity is $371 \mathrm{~km} / \mathrm{s}$. It has an annual sinusoidal period because of the Earth's motion around the Sun with $30 \mathrm{~km} / \mathrm{s}$. It has also a daily sinusoidal period because of the Earth's rotation with $0.5 \mathrm{~km} / \mathrm{s}$.

According to my model from 1997 [1] each process that produces electric photons does create also magnetic photons. The cross-section of magnetic photons in a terrestrial laboratory is roughly one million times smaller than that of electric photons of the same energy. The exact value varies with time and has both the annual and the daily period.

As a consequence, magnetic photons are one million times harder to create, to shield, and to absorb than electric photons of the same energy.

The easiest test to verify/falsify the magnetic photon is to illuminate a metal foil of thickness $1, \ldots, 100 \mu \mathrm{m}$ by a laser beam (or any other bright light source) and to place a detector (avalanche diode or photomultiplier tube) behind the foil. If a single foil is used, then the expected reflection losses are less than 30\%. If a laser beam of the visible light is used, then the absorption losses are less than 15\%. My model [1] has to be considered as falsified if the detected intensity is less than $1.0 \times 10^{-12}$ times the intensity that would be detected if the metal foil were removed and the laser beam would directly illuminate the detector.

The observation of magnetic photon rays would be a multi-dimensional revolution in physics. Its implications would be far-reaching.

(1) The experiment would provide evidence of a second kind of electromagnetic radiation. These magnetic photon rays are more penetratable than electric photon light of the same wavelength. Hence, they may find applications in medicine where X-ray and ultrasonic diagnostics are not useful. X-ray examinations include a high risk of radiation damages, because the examination of teeth requires high intensities of X-rays and genitals are too sensible to radiation damages. Examinations of bones and the brain may also become possible.

(2) The experiment would confirm the existence of a new vector gauge boson, Salam's magnetic photon from 1966. It has the same quantum numbers as Einstein's electric photon, i.e. spin of one, negative parity, zero rest mass, and zero charge.

(3) A positive result would provide evidence of an extension of quantum electrodynamics which includes a symmetrization of Maxwell's equations from 1873.

(4) The experiment would provide indirect evidence of Dirac's magnetic monopoles from 1931 and the explanation of the quantization of electric charge. This quantization is known since Rutherford's discovery of the proton in 1919.

(5) My model describes both an electric current and a magnetic current, even in experimental situations which do not include magnetic charges. This new magnetic current has a larger specific resistance in conductors than the electric current. It may find applications in electronics.

(6) Dirac noticed in 1931 that the coupling constant of magnetic monopoles is much greater than unity. This raises new questions concerning the perturbation theory, the renormalizability, and the unitarity of quantum field theories.

(7) The intensity of the magnetic photon rays should depend on the absolute velocity 
of the laboratory. The existence of the absolute velocity would violate Einstein's relativity principle of special relativity from 1905. It would be interesting to learn whether there exist further effects of absolute motion.

(8) The supposed non-existence of an absolute rest frame was the only argument against the existence of a luminiferous aether. If the absolute velocity does exist, we have to ask whether aether exists and what its nature is.

(9) When in 1925 Heisenberg introduced quantum mechanics, he argued that motion does not exist in this theory. This view is taken also in the Copenhagen interpretation of quantum mechanics formulated in 1927/1928 by Heisenberg and Bohr. The appearance of a velocity operator in my model challenges this Copenhagen interpretation. Mathemati-

cally, the introduction of a velocity (and force) operator means that quantum mechanics has to be described not only by partial but also by ordinary differential equations.

(10) Magnetic photon rays may contribute to our understanding of several astrophysical and high energy particle physics phenomena where relativistic absolute velocities appear and where electric and magnetic photon rays are expected to be created in comparable intensities.

(11) Finally, the other interactions may show similar dualities. The new dual partners of the known gauge bosons would be the magnetic photon, the isomagnetic W- and Zboson, and the chromomagnetic gluons. In 1999 I argued that the dual partner of the graviton would be the tordion [2]. This boson has a spin of three and is required by Cartan's torsion theory from 1922 which is an extension of Einstein's general relativity from 1915.

\section{References}

[1] R. W. Kühne, Mod. Phys. Lett. A 12, 3153 (1997); available online via: http://www.worldscinet.com/journals/mpla/preserved-docs/1240/kuhne.pdf http://xxx.uni-augsburg.de/pdf/hep-ph/9708394

[2] R. W. Kühne, Int. J. Mod. Phys. A 14, 2531 (1999); available online via: http://www.worldscinet.com/journals/ijmpa/14/1416/0160.htm] http://xxx.uni-augsburg.de/pdf/gr-qc/9806026 